\title{
Norois
}

Environnement, aménagement, société

\section{Apport de la cartographie à la gestion locale des ressources renouvelables dans les villages $\mathrm{du}$ district de Rufiji (Tanzanie)}

Cartography for local management of natural resources in villages of the Rufiji District (Tanzania)

Stéphanie Duvail, Rose Hogan, Pili Mwambeso, Revocatus XI Nandi, Richard Elibariki et Olivier Hamerlynck

\section{OpenEdition}

Journals

Édition électronique

URL : https://journals.openedition.org/norois/410

DOI : $10.4000 /$ norois.410

ISBN : 978-2-7535-1544-4

ISSN : 1760-8546

Éditeur

Presses universitaires de Rennes

\section{Édition imprimée}

Date de publication : 1 septembre 2005

Pagination : 51-66

ISBN : 978-2-7535-0218-5

ISSN : 0029-182X

Référence électronique

Stéphanie Duvail, Rose Hogan, Pili Mwambeso, Revocatus XI Nandi, Richard Elibariki et Olivier Hamerlynck, « Apport de la cartographie à la gestion locale des ressources renouvelables dans les villages du district de Rufiji (Tanzanie) », Norois [En ligne], 196 | 2005/3, mis en ligne le 15 décembre 2008, consulté le 13 janvier 2022. URL : http://journals.openedition.org/norois/410 ; DOI : https:// doi.org/10.4000/norois.410

Ce document a été généré automatiquement le 13 janvier 2022.

(c) Tous droits réservés 


\section{Apport de la cartographie à la gestion locale des ressources renouvelables dans les villages $\mathrm{du}$ district de Rufiji (Tanzanie)}

Cartography for local management of natural resources in villages of the Rufiji District (Tanzania)

Stéphanie Duvail, Rose Hogan, Pili Mwambeso, Revocatus XI Nandi, Richard Elibariki et Olivier Hamerlynck

\section{NOTE DE L'ÉDITEUR}

Cet article a été reçu le 4 avril 2005 et définitivement accepté le 27 septembre 2005.

\section{Le contexte de la basse vallée du fleuve Rufiji}

À la descente des plateaux tanzaniens, le Rufiji, principal fleuve de Tanzanie, a construit une large plaine alluviale (fig. 1) exploitée par les populations Warufiji ${ }^{1}$ qui y pratiquent une agriculture de décrue associant riz, maïs et légumineuses. Une série de terrasses à sables grossiers surplombe cette plaine inondable. Le paysage dominant de ces terrasses aux sols peu fertiles est celui d'une forêt claire à Brachystegia sp. et Julbernardia sp. (miombo), alternant, lorsque les conditions hydrogéologiques le permettent, avec des îlots de forêt dense, lambeaux de l'ancienne forêt côtière (coastal forest) d'Afrique de l'Est (Burgess et Clarke, 2000). Vers l'est, le fleuve Rufiji très chargé en sédiments, débouche sur l'une des plus grandes mangroves de l'Afrique de l'Est (500 $\mathrm{km}^{2}$ ). 
2 Entre 1968 et 1974 , le district du Rufiji a servi de zone-test pour la politique de villagisation (Ujamaa) de Julius Nyerere.Les Warufiji qui vivaient de manière dispersée sur les levées du fleuve (Telford, 1929) ont été forcés de quitter la plaine inondable et ont été regroupés dans des villages sur les terrasses, selon un plan de villagisation. Abandonnant leurs traditionnelles cultures de décrue, ils ont été contraints de défricher les abords de leurs nouveaux villages et de convertir leurs économies aux cultures de rente (noix de cajou, coton, sésame) et aux cultures de manioc et de sorgho pour l'alimentation.

3 Les agriculteurs ne se sont que très partiellement adaptés à ce nouveau milieu et face aux mauvais résultats économiques de ce programme de villagisation dans le Rufiji, nombre de familles sont retournées cultiver les terres fertiles de la plaine inondable à partir des années 1980. Cependant, au regard de la loi, ce retour est illégal. Les populations de la plaine inondable et du delta (environ 150000 personnes) se trouvent donc dans une situation d'insécurité foncière et économique.

Figure 1 : Localisation de la basse vallée du Rufiji

Location of the lower Rufiji floodplain

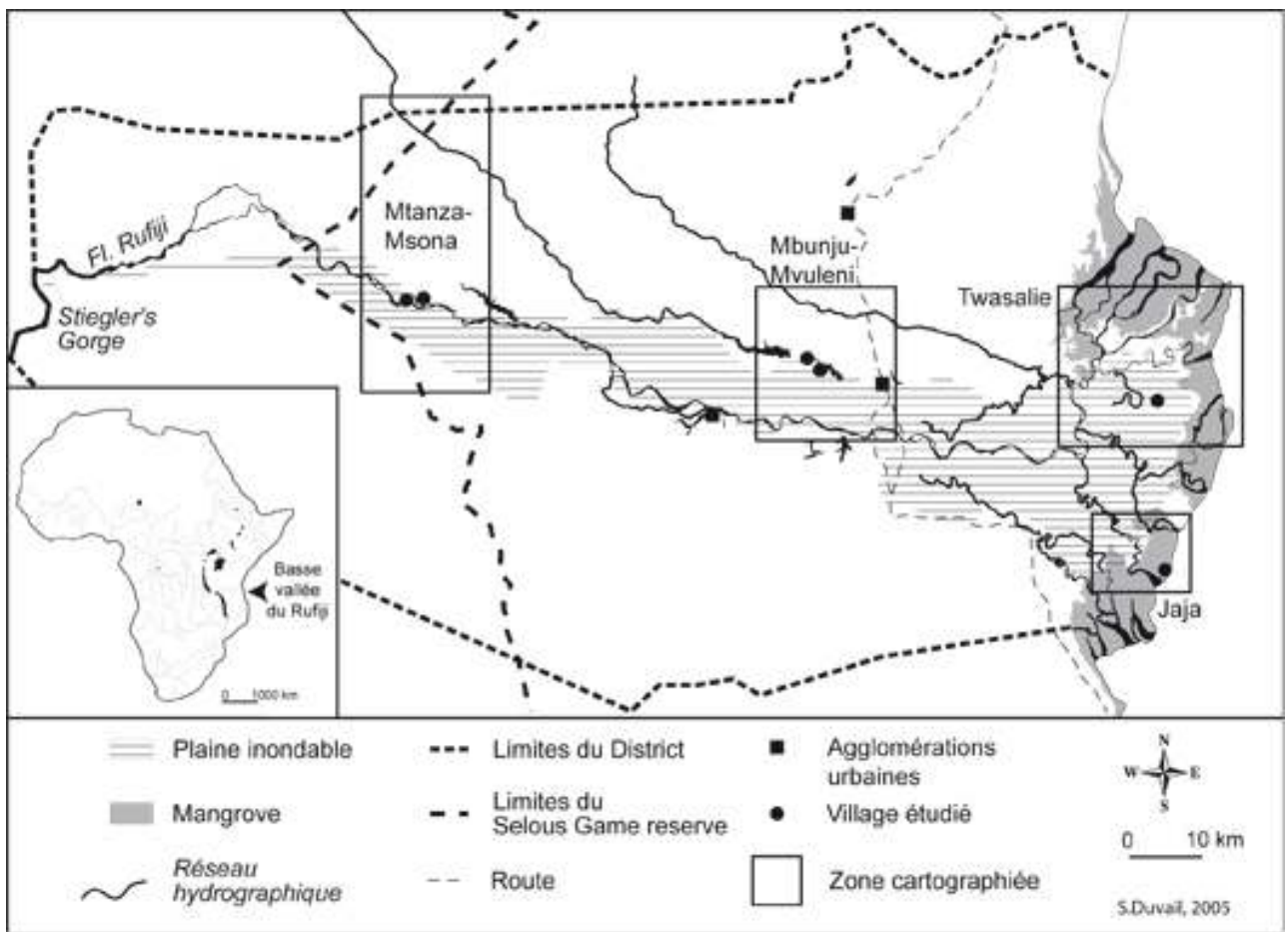

Cet espace autrefois d'accès difficile et mal connecté au reste de la Tanzanie, est en pleine mutation. Une série d'événements, dont les effets se conjuguent, augmente cette insécurité foncière et est à l'origine d'une pression accrue sur les ressources renouvelables.

La construction d'une route reliant la capitale économique Dar Es Salaam au Sud Tanzanien et plus particulièrement du pont du Rufiji (mis en service depuis 2001) a amélioré l'accès au Rufiji et son intégration aux marchés national et international. Cet aménagement a des effets positifs sur les économies locales (écoulement des produits, accès facilité à des biens de consommation) mais rend également la basse vallée du Rufiji attractive pour des acteurs extérieurs. Dans le district du Rufiji, l'un des plus boisés de Tanzanie, la pression sur les ressources forestières était déjà très forte en 
dépit d'un accès routier difficile : au cours des 15 dernières années, de nombreuses espèces d'arbres ${ }^{2}$ autrefois abondantes sont devenues très rares ou ont atteint leur seuil d'extinction commerciale (John et Hamerlynck, 2003). Cette dégradation du couvert forestier est liée aux pratiques des petits producteurs de charbon mais aussi et surtout à des prélèvements illégaux de bois, par des opérateurs économiques basés à Dar Es Salaam. Depuis la construction de la nouvelle route et du pont, le taux d'exploitation des ressources forestières, déjà élevé, a considérablement augmenté sous l'effet de la pression de nouveaux agents économiques exogènes, liés au marché international (Milledge et Kaale, 2004). En 2004, le bois était illégalement exporté par camions de 40 tonnes vers le port de Dar Es Salaam en direction des marchés asiatiques et en particulier de la Chine ou bien traité sur place dans l'une des dix grandes scieries construites depuis l'ouverture du pont. La construction de cette route et, de façon concomitante, la commercialisation de protections sanitaires contre la trypanosomiase, précédemment fatale aux troupeaux bovins, ont aussi eu pour conséquence l'arrivée de nouveaux acteurs (éleveurs Barbaig et Sukuma venus du Nord de la Tanzanie), à l'origine de conflits d'usage nouveaux entre agriculteurs et éleveurs (Mwilawa, 2003).

6 Le projet de construction d'un barrage hydro-électrique sur le site de Stiegler's Gorge, laisse également présager d'importantes modifications à moyen terme. Cet ouvrage, destiné à produire de l'hydro-électricité pour une exportation vers l'Afrique australe, atténuera notamment les pointes de crue, pourtant essentielles pour l'agriculture de décrue dans la plaine inondable (riz, maïs et légumineuses), pour la pêche et pour le maintien des fonctions des écosystèmes de mangrove. Il est également prévu qu'en aval du barrage, 85000 hectares de la plaine inondable seront aménagés pour une agriculture irriguée. Un tel projet, conçu dans les années 1970, période à laquelle les Warufi avaient été déplacés vers les terrasses, impliquerait un aménagement très coûteux (estimé à 1 milliard de dollars US en 1980 soit approximativement 2,3 milliards de dollars US en 2004), difficile à rentabiliser (Agrar-Und Hydrotechnik GMBH, 1982) et qui aurait des impacts socio-économiques discutables pour les populations Warufiji.

7 Parallèlement à ces mutations actuelles et à venir, la publication d'une série de nouvelles lois de décentralisation des services de l'État tanzanien permet aux communautés locales de gérer les ressources renouvelables à l'échelle villageoise. Un projet d'éco-développement, le Rufiji Environmental Management Project (REMP) financé par la coopération néerlandaise, et mis en œuvre par le district du Rufiji avec un appui technique de l'UICN (Union Mondiale pour la Nature) a démarré en 1998. Il s'est donné pour tâche de faciliter un transfert vers les villages des droits de gestion des ressources renouvelables (forestières, cynégétiques, halieutiques) auparavant appartenant à l'État. Ces transferts de gestion sont réalisés dans le cadre de la rédaction, en commun avec les villageois, de plans de gestion de l'environnement villageois (VEMP: Village Environmental Management Plan).

8 La rédaction d'un tel plan de gestion nécessitait que soit mieux connu et délimité l'espace du village. Les gestionnaires du projet REMP ont donc fait appel à notre équipe de recherche franco-tanzanienne pour réaliser une cartographie du terroir tel qu'il est utilisé par les habitants du village. Cet exercice, en apparence simple, se heurtait à un certain nombre d'écueils.

9 Premier écueil, un important décalage existe entre le terroir villageois officiellement attribué lors de la période Ujamaa et le terroir actuellement utilisé par les populations Warufiji. Officiellement, seuls les champs sur les terrasses à miombo appartiennent aux 
villages. Les terroirs traditionnels de la plaine inondable n'ont pas d'existence administrative, pour des raisons historiques, s'agissant d'un espace d'habitation interdit à l'époque Ujamaa, mais aussi en raison de la méconnaissance qu'ont les agents de l'État des pratiques agricoles des paysans du Rufiji : les systèmes traditionnels ne sont pas jugés dignes d'intérêt par une administration qui a une approche normative de l'agriculture. Ce discrédit est renforcé par la très faible représentation des populations Warufiji au sein de l'administration du district du Rufiji. Les taux de scolarisation dans le district du Rufiji sont très faibles : seuls $0,75 \%$ des enfants Warufiji entrent à l'école secondaire (Rufiji District Council, 1997). Ces faibles taux de scolarisation sont eux aussi liés à l'héritage de la période Ujamaa et à la non-reconnaissance des modes de vie actuels de la paysannerie Warufiji, les écoles étant toujours situées sur les terrasses, au centre des villages Ujamaa tandis que les familles vivent dans la plaine inondable pendant la saison agricole (octobre à juin).

Dans un contexte de décentralisation des services de l'État, les agents du district ont vu récemment se renforcer leurs compétences en matière d'agriculture, de gestion des ressources naturelles et d'éducation mais sans disposer ni de données objectives sur l'économie paysanne, ni de moyens de se déplacer dans les villages avant l'existence du projet REMP, dont l'un des objectifs affichés est de favoriser la communication entre gestionnaires du district et populations locales.

11 Cette conjonction de facteurs (discours normatif hérité de l'Ujamaa, méconnaissance des modes de vie traditionnels et actuels des populations) explique la persistance d'un mythe d'une plaine vide d'hommes et la permanence de rapports officiels considérant que les espaces de la plaine inondable sont sous ou inutilisés. Tout cela est contredit par l'observation des paysages de la basse vallée (photo 1), marqueterie de champs et d'habitations. En raison de ce décalage entre les informations officielles et la réalité, l'un des premiers objectifs de cette cartographie était alors de définir l'espace fonctionnel utilisé actuellement par les communautés.

Photo 1 : Plaine inondable du Rufiji au sud d'lkwiriri (cl. S. Duvail, 2004)

(Rufiji Floodplain, south of Ikwiriri)

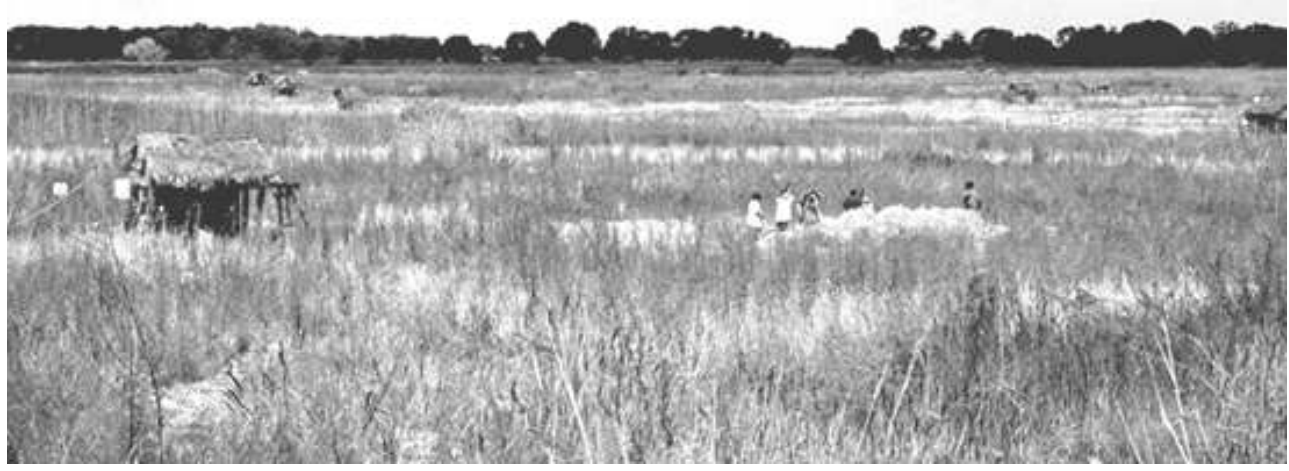


12 Second écueil, l'exercice est compliqué par le fait que, si le village est l'entité administrative de base, il recouvre parfois une réalité sociologique plus complexe qui est celle d'un regroupement plus ou moins lâche de groupes familiaux. En effet, la politique d'Ujamaa a eu pour conséquence de réunir plusieurs lignages au sein de nouveaux villages. La solidité relative de ces créations administratives se mesure aux mouvements centrifuges qui se manifestent parfois avec la création de nouveaux hameaux (sub-village). Certains des villages créés ex-nihilo par la politique de villagisation ont même disparu à partir des années 1980, souvent en raison d'une mauvaise localisation géographique et d'un accès difficile à une source d'eau potable. Ces disparitions de villages ont donné lieu à des retours de population vers leurs terroirs d'origine, sans que ce retour soit enregistré officiellement. Au terme de ces recompositions et en dépit de cette complexité sociologique, on peut cependant considérer que les villages restants sont des entités administratives relativement solides, dont les institutions sont réinvesties par les autorités traditionnelles. En effet, si le projet socialiste d'une agriculture fortement mécanisée a été un échec économique, en revanche les populations Warufiji ont pu trouver certains intérêts à être regroupées au sein de villages : elles bénéficient des services de l'État en matière de santé et de la desserte assurée par la route, qui a aujourd'hui supplanté le fleuve comme voie de communication principale. De plus, dans la basse vallée du fleuve, les villages Ujamaa ont été implantés à proximité de lacs d'eau douce. La pêche qui y est pratiquée contribue aujourd'hui à hauteur de $30 \%$ au budget des ménages (Turpie, 2000). L'exportation du poisson fumé-séché vers les agglomérations nouvelles autour de la route, vers Dar Es Salaam et vers les grandes villes du sud apporte même près de $65 \%$ des revenus monétaires (Turpie, 2000). Ces lacs représentent également une importante source d'eau domestique et potable. Pour ces mêmes villages en bordure de la plaine inondable, la relocalisation a rapproché les communautés locales des forêts qu'elles exploitaient déjà mais au prix de longues marches. Nombre d'habitants ont ainsi deux maisons : l'une, souvent bâtie sur pilotis, est située dans la plaine inondable et est occupée une majeure partie de l'année, l'autre est bâtie en dur dans le village ou hameau et utilisée trois mois de l'année, en dehors des périodes agricoles ou par les membres de la famille qui ne sont pas ou plus en âge de travailler dans les champs. Cette double localisation permet la mise en œuvre d'une large palette d'activités agricoles et extractivistes.

Enfin une dernière difficulté et non des moindres, a été l'absence d'information cartographique utilisable pour la région du Rufiji. Il n'existait pas de document à l'échelle du district pouvant servir de fond cartographique pour la constitution d'un Système d'Information Géographique. Les quelques documents existants étaient très schématiques ou spatialement incohérents, comme dans le cas de la carte réalisée par le Tanzania's Natural Resources Information Centre (TANRIC) sur la base de photographies aériennes non ortho-rectifiées (TANRIC, 2001). D'autres documents existants font une description erronée des ensembles paysagers : ainsi en est-il par exemple des paysages décrits par la carte de Hunting Technical Services, (1996).

14 Au-delà d'un simple exercice cartographique, les objectifs étaient donc, dans un premier temps, de contribuer à une meilleure description des paysages et de l'occupation de l'espace de la basse vallée du Rufiji et, dans un second temps, de participer à un processus de planification environnementale basé sur un dialogue entre 
communautés locales, gestionnaires et chercheurs autour d'une carte qui soit mieux représentative de l'actuelle organisation de l'espace de la basse vallée du Rufiji.

\section{La démarche de planification environnementale et les méthodes de cartographie}

L'exercice de cartographie s'est inscrit dans un processus plus large de planification environnementale réalisée en collaboration entre les gestionnaires (fonctionnaires du district et conseillers techniques du projet REMP), les populations de 4 villages candidats au transfert des droits en matière de gestion forestière et les chercheurs (tanzaniens et européens). Ce processus s'est appuyé sur une modification de la loi tanzanienne qui vise à décentraliser les droits de gestion des forêts mais a pour objectif plus large de réduire le décalage existant entre les droits coutumiers des communautés locales et la définition officielle du village.

\section{Le processus de planification environnementale à l'échelle villageoise}

16 Une première étape a consisté à établir une relation de confiance entre les différents protagonistes (discussions, organisation d'ateliers d'information sur les nouvelles lois, clarification des rôles respectifs des différentes institutions d'État). Au terme de cette phase, assez longue, quatre des onze villages contactés ont souhaité participer à l'exercice de planification environnementale.

Afin d'éviter une multiplication des institutions, le processus s'est appuyé sur les structures et les règles déjà existantes au sein du village. Depuis le Local Government Authorities Act de 1982, l'Assemblée villageoise (Village Assembly Meeting ou Kikao Cha Hadhara), composée de tous les habitants du village âgés de plus de 18 ans, est l'organe statutaire en charge de l'élection d'un Conseil municipal de 25 personnes (Village Government ou Halmashauri ya Kijiji), présidé par un maire (Chairman ou Mwenyekiti) (Mniwasa et Shauri, 2001). La direction du processus de planification environnementale a été confiée à ce conseil municipal. De plus, il existe au sein de chaque village, des Comités villageois (Village commitee ou Kamati), ayant un rôle de conseil puis d'exécution des décisions prises par le Conseil municipal en matière de finance, de pêche, de sécurité, etc. Aussi, un Comité villageois de l'Environnement (Village Environment Commitee ou Kamati Ya Maliasili na Mazingira) a-t-il été créé.

- Dans un premier temps, l'Assemblée du village s'est réunie. L'historique du village, l'origine des familles a été retracée par les anciens et le territoire traditionnel des groupes familiaux composant le village a été décrit, à l'aide de maquettes réalisées au sol à partir de terre et de branchages. Les différentes activités économiques et les modes d'exploitation des ressources naturelles ont été détaillés. Lorsque le groupe s'accordait sur une version, elle était transcrite en Swahili par un secrétaire. Parallèlement à ce processus, des enquêtes socioéconomiques et anthropologiques ont été menées par les chercheurs tanzaniens et européens. Compte tenu de la complexité des relations sociologiques au sein des villages, une attention particulière a été portée à l'analyse de la communication interne au village. Des « cartes de confiance ", représentant les réseaux d'information des individus ont été réalisées et ont permis de comprendre les relations entre les différents groupes. 
- Dans un second temps, des techniques de gestion participative ont été utilisées pour la conception d'un plan de gestion selon les étapes suivantes : analyse des problèmes par l'Assemblée du village, hiérarchisation des priorités, recherche de solutions et des potentialités de développement pour le village. Plusieurs mois, voire années, ont été consacrés à ces étapes afin de permettre aux différentes communautés composant le village de s'accorder sur une vision de l'aménagement de leur territoire. Au terme de cet exercice, un zonage du territoire a été défini ainsi qu'un règlement (bylaws) définissant les droits d'accès et d'usage des ressources renouvelables, en particulier forestières. Ce zonage a été soumis à l'Assemblée du village et devait recueillir $75 \%$ des votes pour être adopté. La nouvelle loi sur les forêts le permettant, une partie de la forêt a été enregistrée comme réserve forestière villageoise : elle comporte une zone ouverte à l'extraction de bois et une zone de réserve intégrale au sein de laquelle aucune coupe ne sera pratiquée pendant 10 années. Des patrouilles villageoises en assurent le contrôle. Selon la loi, 30 \% des amendes revient au village (70 \% au district) en cas de coupe illégale.

- Dans un quatrième temps, une cartographie du village a été produite. La réserve forestière villageoise a été choisie par les villageois puis délimitée par une équipe de topographes du Service National des Forêts. En vue de la production d'une carte synthétique, le territoire villageois a été arpenté, les paysages ont été décrits et les différentes occupations de l'espace ont été relevées par une équipe multi-institutionnelle (villageois/agents du district/ chercheurs) munie de systèmes GPS. Au terme de la production de cartes pour chacun des villages, les résultats (plan de gestion en Swahili et cartes) ont ensuite été discutés au sein des villages avant d'être approuvés ou amendés. Les règlements ont ensuite été soumis pour approbation au district, et la réserve forestière enregistrée au niveau national.

- Enfin, un appui à la mise en œuvre des plans de gestion villageois est fourni par le projet REMP avec une formation à la gestion, une mise en place de patrouilles de surveillance villageoise, une aide à l'amélioration des transports et communication (par exemple l'achat d'un bateau et d'une radio pour les villages du delta).

\section{Les méthodes utilisées pour la cartographie du village} capteur Thematic Mapper du satellite Landsat 5 d'une résolution au sol de $30 \times 30 \mathrm{~m}$, datant de 2000 et analysées en complémentarité d'une série de photographies aériennes $^{3}$ de 1999 au 1/50 000 que nous avons préalablement ortho-rectifiées.

Pour la réalisation de chaque Système d'Information Géographique, la chaîne méthodologique a comporté les étapes suivantes :

- Préparation des documents satellitaire

- Numérisation des photographies aériennes (250 au total).

- Relevés sur le terrain de points de calage (5 par photographie aérienne) avec un GPS d'une précision de 10 à $30 \mathrm{~m}$. Les carrefours de pistes, bâtiments remarquables ou bien, dans la mangrove, les confluences de marigots ont été privilégiés.

- Ortho-rectification des photographies aériennes avec le logiciel Erdas Imagine.

- Pré-traitement et analyse de l'image satellitaire enregistrée en 2000.

- Analyse de paysages

- Classification des types de paysages en fonction de leur degré d'hétérogénéité spatiale sur l'image et sur les photographies aériennes.

- Analyses paysagères réalisées sur le terrain par une équipe composée d'un géographe, d'un botaniste, d'un écologiste et d'habitants du village. 
- Etablissement d'une correspondance entre l'organisation paysagère telle que relevée au sol et les catégories établies à partir des documents satellitaires.

- Cartographie des usages de l'espace

- Visite de terroirs par une équipe multi-institutionnelle (villageois, agents du district, chercheurs) munie de systèmes GPS, relevés des toponymies, établissement d'une typologie des usages de l'espace.

- Entretiens non directifs avec les usagers.

- Production des documents

- Intégration des données dans un Système d'information Géographique (Arc View d'Esri).

- Production de cartes d'occupation de l'espace villageois à l'échelle du 1:50000.

- Impression.

- Restitution auprès de l'Assemblée du village et correction éventuelle.

- Édition.

\section{Les résultats : Description paysagère et production de quatre plans de gestion de l'environnement villageois}

Au terme de cinq années (1999-2003), quatre plans de gestion villageois ont été produits selon le processus décrit, édités en Swahili puis traduits en anglais (Jaja Village, 2004 ; Mtanza-Msona Village, 2004 ; Mbunju-Mvuleni Village, 2004 ; Twasalie Village, 2004). Les décisions de gestion environnementales ont été validées par les services de l'État et les réserves forestières villageoises ont été enregistrées au niveau national. La cartographie proprement dite et la constitution de Systèmes d'Information Géographique pour ces quatre villages ont nécessité sept mois de travail. Deux plans de gestion concernent des villages de la plaine inondable (Mtanza-Msona et MbunjuMvuleni) et deux des villages du delta (Twasalie et Jaja) (fig. 1), avec des résultats différenciés en fonction de la situation foncière et sociologique initiale du village.

\section{Mtanza-Msona (fig. 2)}

Le village de Mtanza-Msona comptait 1830 personnes selon les chiffres du dernier recensement (National Bureau of Statistic, 2003). L'ethnie Ndengereko (branche Wahuringo) y est majoritaire, et coexiste avec des groupes Matumbi, Pogoro, Hehe et Zaramo. Le terroir du village s'étend de part et d'autre du fleuve Rufiji. La rive sud est habitée et cultivée selon la micro-topographie et la diversité pédologique des sols. Les sols de mbawila (sols argileux des dépressions) sont considérés comme les meilleurs sols. Les levées sablo-argileuses sont matérialisées par une végétation de manguiers et d'acacias, témoin de leur occupation dans la période pré-Ujamaa. Les maisons actuelles sont construites sur pilotis dans les champs individuels. De nombreuses familles ont cependant une seconde maison dans le village 'officiel' au nord du fleuve. Une agriculture pluviale est aussi pratiquée dans les cuvettes argileuses situées au nord du village. Les deux hameaux principaux du village (Mtanza et Msona) sont positionnés sur la route Ikwiriri-Mloka et de part et d'autre d'un lac de petite taille. Le paysage dominant des terrasses de la rive nord est celui d'une forêt claire sur sols brun-rouge sableux, alternant avec des étendues de savane arborée à herbeuse dans les dépressions plus argileuses. Cette forêt claire, facilement accessible depuis Dar Es Salaam par une 
piste créée lors de travaux de prospection pétrolière, est soumise à une forte exploitation.

Figure 2 : Carte du village de Mtanza-Msona Mtanza-Msona village map

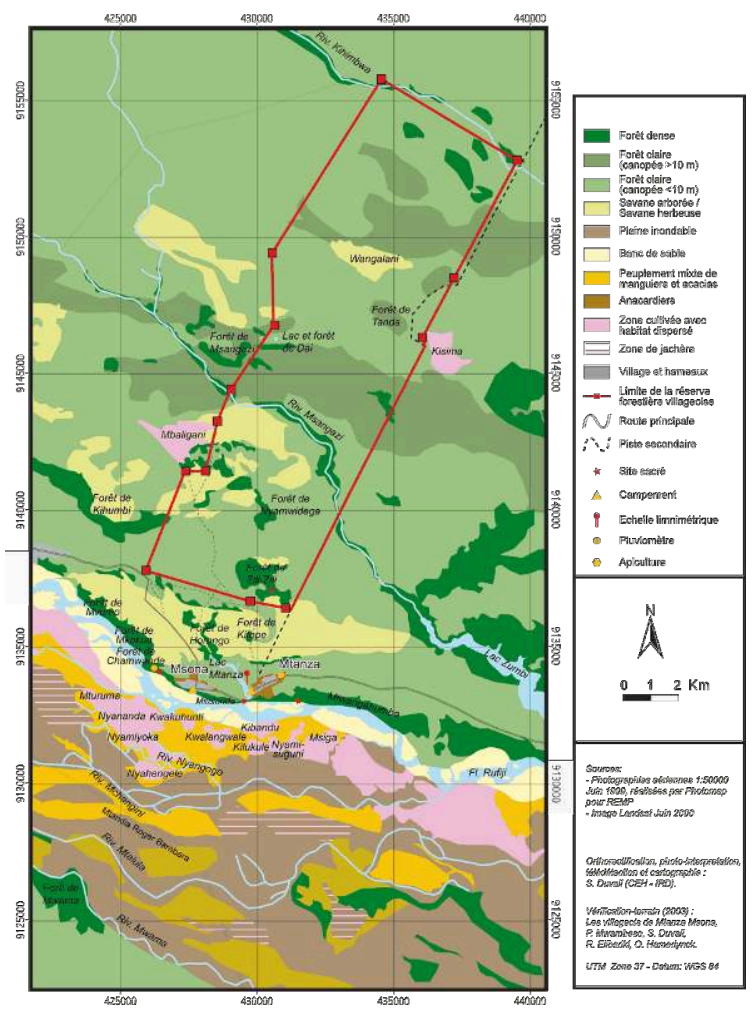

22 Le village est en conflit avec la grande réserve de chasse du Selous $\left(48000 \mathrm{~km}^{2}\right)$. Outre le fait que le village conteste la limite sud de l'aire protégée, mitoyenne au village, la faune de la Réserve représente un très grand danger pour les agriculteurs : ainsi entre août 2002 et avril 2004, 35 personnes ont été tuées par un lion mangeur d'hommes dans la partie sud de la plaine inondable (Baldus, 2004). Compte tenu de ce conflit avec l'Aire Protégée, les villageois se sont montrés très suspicieux à l'égard de toute proposition de plan de gestion 'environnementale'. Un atelier d'information sur les possibilités des nouvelles lois forestières a toutefois été organisé dans le village par une équipe de juristes tanzaniens (LEAT : Lawyers' Environmental Action Team), et l'option de posséder un droit de gestion de la forêt a fortement intéressé les habitants (Majamba et al., 2001). $\mathrm{Au}$ bout de cinq mois de réflexion, le village a accepté de devenir l'un des quatre villages pilotes du projet.

Une première maquette au sol du terroir villageois a été produite en août 1999. La discussion autour du plan a duré une année et une première version a été rédigée en septembre 2000. L'Assemblée a décidé de classer « réserve forestière villageoise » une large superficie au nord du village comprenant les forêts galeries autour de la rivière Msangazi et les principales forêts sacrées du village (notamment la forêt de Zili-Zili). La zone d'usage réservée aux villageois entoure le village et s'étend à l'ouest de cette réserve villageoise. Une patrouille de surveillance de la forêt a été créée et a procédé à de premières arrestations de trafiquants de bois en mars 2001. 
24 En dépit de la méfiance initiale, le plan de gestion environnementale semble avoir favorisé une dynamique économique locale. Les étapes de la planification ont permis d'améliorer les relations entre les gestionnaires locaux du district et les villageois. Un accord entre les deux hameaux villageois a été trouvé pour la gestion des pêches sur le lac, comprenant un accord sur fermeture à la pêche du lac pendant les deux mois de crue afin de favoriser la reproduction des géniteurs. Autre façon d'améliorer la productivité du lac, un système de Misaka-saka a été testé avec succès : il s'agit d'un enchevêtrement de branchages disposés dans l'eau, servant à la fois d'abri, de lieu de ponte et de frayère artificielle de poissons, adaptation du principe des Akadja utilisées dans les lagunes du Bénin. Des revenus monétaires sont générés par la levée d'une taxe pour les pêcheurs non-résidents et par la vente du bois de la partie de la réserve forestière réservée à la coupe. Enfin un processus de révision de la frontière du Selous a été initié avec l'appui des autorités du district et du projet REMP.

\section{Mbunju-Mvuleni (fig. 3)}

Le processus n'a pas rencontré un tel succès dans le village de Mbunju-Mvuleni. Bien qu'il s'agisse d'un plus petit village (590 personnes au dernier recensement; National Bureau of Statistics, 2003), d'origine Ndengereko (75 \%) et Ngindo (25\%), le village est divisé en cinq hameaux: trois sont implantés sur les terrasses du fleuve (Mbunju, Mvuleni, Mpima) et deux sur les levées du fleuve dans la plaine inondable (Kilalani, et Mupi). Comme à Mtanza-Msona, la pêche dans les lacs de Uba et Ruwe est une importante source de revenu et est pratiquée en complémentarité d'une agriculture pratiquée sur les sols très fertiles de la plaine inondable, située cette fois au nord du fleuve. Une partie du terroir villageois a été aménagé dans les années 1980 par un projet iranien et les terres concédées à des agriculteurs d'Ikwiriri. Les périmètres sont aujourd'hui abandonnés mais le gouvernement prévoit une réhabilitation.

Une cartographie fine et un travail de reconnaissance paysagère détaillée sur le terrain ont permis de préciser l'organisation d'un paysage forestier mal connu: souvent décrite comme une zone de miombo uniforme, la partie nord du village est en fait caractérisée par une mosaïque de forêts denses, de forêts claire et de savane arborée à herbeuse. À l'est du village, une vaste zone de foret dense, la forêt de Ngumburuni, a récemment été décrite (Durand, 2003). La partie nord du village comprend des lambeaux de cette forêt. Vers l'ouest, le paysage est celui d'une mosaïque complexe de petits îlots de forêt dense dans les dépressions où se concentrent les écoulements, et de savanes herbeuses dans les zones les plus hautes. Cette organisation particulière du paysage est génératrice d'une biodiversité plus élevée que celle du miombo environnant. La cartographie a également permis de mettre en évidence le très fort taux d'occupation des sols de la plaine inondable, et en particulier l'existence d'un système de jachère avec un rythme de rotation de 3 à 10 ans en fonction de la qualité des sols. 
Figure 3 : Carte du village de Mbunju-Mvuleni Mbunju-Mvuleni village map

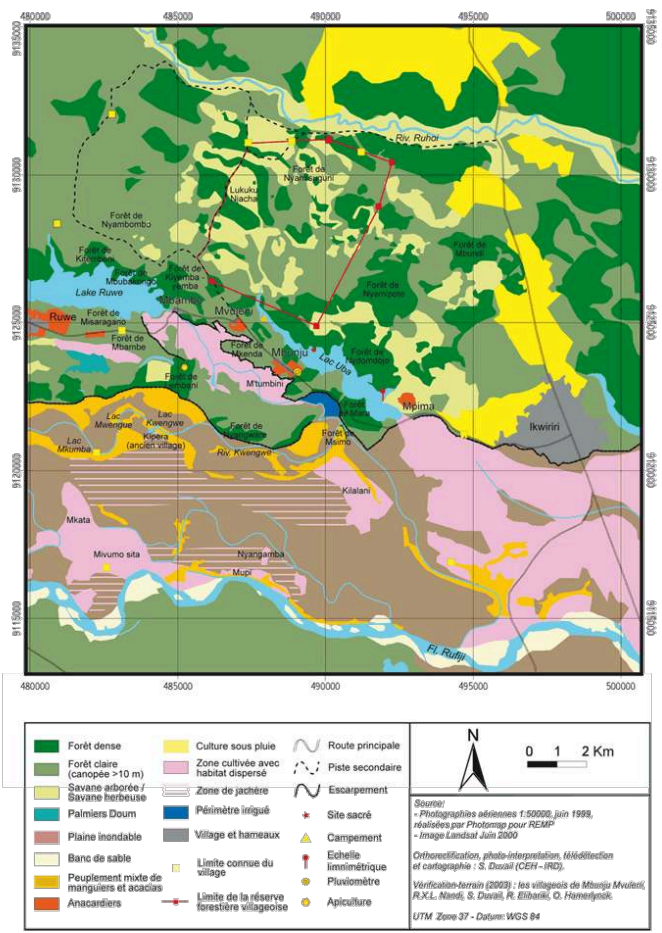

Dans ce village proche de la route vers Dar Es Salaam-Mtwara et du nouveau pont du Rufiji, la pression sur les ressources forestières et foncières est forte et les villageois ont tout de suite été intéressés par un outil juridique capable de sécuriser leurs droits sur la forêt et sur leurs terres. Cependant le processus a été fortement ralenti par les dissensions entre les différents hameaux. Les hameaux de Mbunju, Mvuleni et Kilalani souhaitaient exclure les hameaux de Mupi et de Mpima des assemblées plénières car leurs habitants, bien qu'enregistrés dans le même village au cours de la villagisation, ne se sont installés dans la zone que depuis deux générations. Qui plus est, il existe un conflit entre les hameaux localisés sur les terrasses (Mvuleni, Mbunju et Mpima) sur la gestion des pêches du lac Uba. Les zones de pêche du lac Ruwe sont également partagées avec les deux villages voisins, situation potentiellement conflictuelle.

Un consensus a pu être trouvé sur la création d'une réserve forestière qui a permis de diminuer localement le flux de l'export illégal du bois et de générer des revenus monétaires pour le village grâce aux amendes et à la vente du bois par les villageois eux-mêmes. Comme à Mtanza-Msona, le tracé de la réserve villageoise a été choisi de façon à y inclure les forêts sacrées. D'autres activités économiques ont eu un effet positif (apiculture, diversification des produits de maraîchage, vaccination des poulets contre la maladie de Newcastle, diffusion de foyers améliorés) mais aucun consensus n'a pu être trouvé sur la gestion des pêches dans les lacs.

\section{Twasalie (fig. 4)}

Dans le delta, Twasalie est également un village issu du programme de villagisation et composé de plusieurs hameaux. 890 habitants, en majorité Nyagatwa, se répartissent sur une dizaine de sites, constituant officiellement 5 hameaux implantés sur des 
massifs dunaires. L'histoire orale fait remonter la présence de ces populations, aujourd'hui musulmanes, à environ 2000 ans. Les familles sont dispersées mais la cohabitation entre les différents groupes ne semble pas pour autant conflictuelle, en partie parce que le programme de villagisation n'a donné lieu a aucun déplacement de population.

Figure 4 : Carte du village de Twasalie Twasalie village map
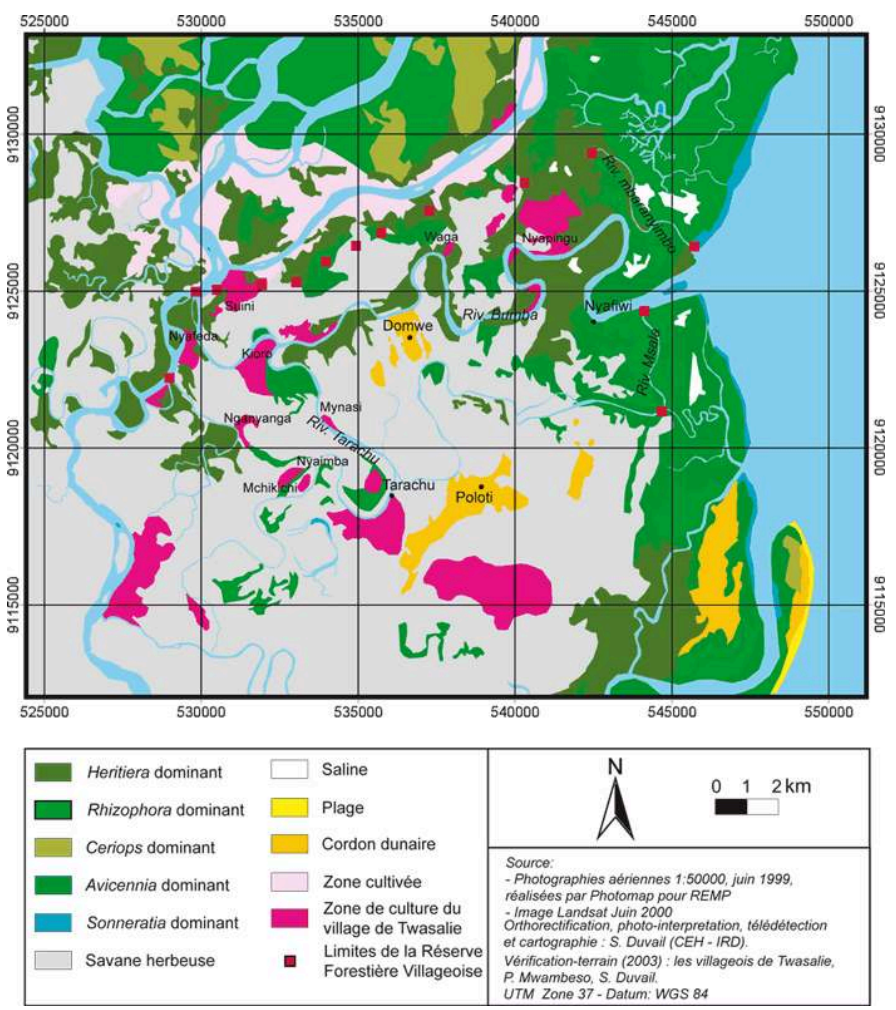

La cartographie a permis de mettre en évidence une organisation de terroir basée sur la micro-topographie et le cycle des marées : les meilleurs champs se trouvent à proximité du fleuve et sont inondés par des eaux douces lors des marées de vives eaux. Sur ces terroirs de jongoni, deux à trois récoltes de riz par an sont possibles tandis qu'une seule récolte de riz est réalisée dans les parties les plus hautes des plaines inondables du delta. La pêche estuarienne et côtière sont des activités importantes, la pêche crevettière en étant l'activité la plus rémunératrice. L'exploitation des ressources des mangroves représente également une part non négligeable du revenu des ménages (collecte de bois de chauffe et de construction, production de miel). Enfin une production de sel est réalisée dans les salines (jangwa).

Dans les années 1990, dans un contexte de libéralisation de l'économie tanzanienne, un projet de ferme crevetticole a vu le jour: un investisseur privé (l'African Fishing Company) planifiait de créer une ferme de 10000 hectares dans le delta du Rufiji, qui aurait annexé une partie du village de Twasalie. Le projet, financé sur crédits européens, a donné lieu à des études d'impact contradictoires : deux études d'impact environnemental commanditées par l'African Fishing Company sont publiées en 1996 et 1997, toutes deux favorables au projet tandis qu'une contre-expertise de la National Environmental Management Commission (NEMC), organe consultatif, placé sous l'autorité de la Vice-Présidence de l'État tanzanien, donne un avis négatif argumentant que les 
chiffres des bénéfices prévus par les premières études d'impact sont gonflés et que le delta est plus productif à l'état naturel. Le rapport remet aussi en cause le montage financier et montre en particulier que des compensations pour les populations ne sont pas prévues dans le budget. Enfin, l'étude émet des doutes sur l'opportunité d'importer des armes et des munitions pour le projet (Masalu, 2003). Malgré les réserves émises par le rapport de NEMC, le gouvernement approuve le projet en novembre 1997. Cette décision a généré la protestation et l'opposition à la fois des communautés locales, de plusieurs services de l'État (département de l'Énergie et des Mines, département des Forêts, département du Tourisme), des ONG environnementalistes, du Parc marin de l'île de Mafia nouvellement créé, et du projet REMP (Rufiji Environmental Management Project) en phase de démarrage. Un procès a été intenté devant la Haute Cour de Justice tanzanienne par les communautés villageoises assistées par LEAT (Lawyer's Environment Action Team) pour le motif invoqué suivant: "Harm to life and livelihood of coastal communities through its destructive activities. » Depuis, ce projet de crevetticulture est en suspens. La Haute Cour de Justice ne se prononçant pas, le projet est gelé et les bailleurs de fonds se sont désengagés.

Twasalie fait partie des villages qui ont porté plainte contre ce projet de ferme crevetticole. Une autre source d'inquiétude pour les villages de Twasalie est liée à l'arrivée de migrants en provenance des collines avoisinantes s'installant dans la mangrove en la défrichant pour une riziculture itinérante. Dans ce contexte, le projet de planification environnementale et de cartographie du territoire villageois a été très rapidement adopté par l'Assemblée du village. Les priorités d'un tel plan, tels que définies par le village ont été : l'établissement de règles d'utilisation des ressources naturelles, une reconnaissance des frontières du village, l'amélioration des conditions d'agriculture et d'élevage, le désenclavement du village. Un plan de gestion villageois était prêt dès la mi-février 2000 comprenant un zonage et un règlement. Le projet REMP a équipé le village d'un système de radio HF et d'un bateau à moteur pour l'organisation de patrouilles et pour les évacuations en cas d'urgence médicale.

\section{Jaja (fig. 5)}

Jaja est un village d'implantation ancienne n'ayant fait l'objet d'aucun programme de villagisation. 1500 habitants, à majorité Ndengereko, s'y répartissent en trois hameaux, tous implantés sur un grand cordon littoral sableux, planté de cocotiers, d'anacardiers et de manguiers. Il s'agit d'un village peu tourné vers l'agriculture et davantage vers l'exploitation des plantations de cocotiers, les chantiers de construction de bateaux, et surtout la pêche crevettière destinée à l'exportation. Dans ce domaine, les pêcheurs de Jaja subissent la concurrence de chalutiers semi-industriels qui pêchent très souvent à proximité des côtes. Comme à Twasalie, la mangrove est une importante source de revenu et le choix a été fait par le village de déclarer toute la zone de mangrove au nord du village comme réserve forestière villageoise. 
Figure 5 : Carte du village de Jaja Jaja village map

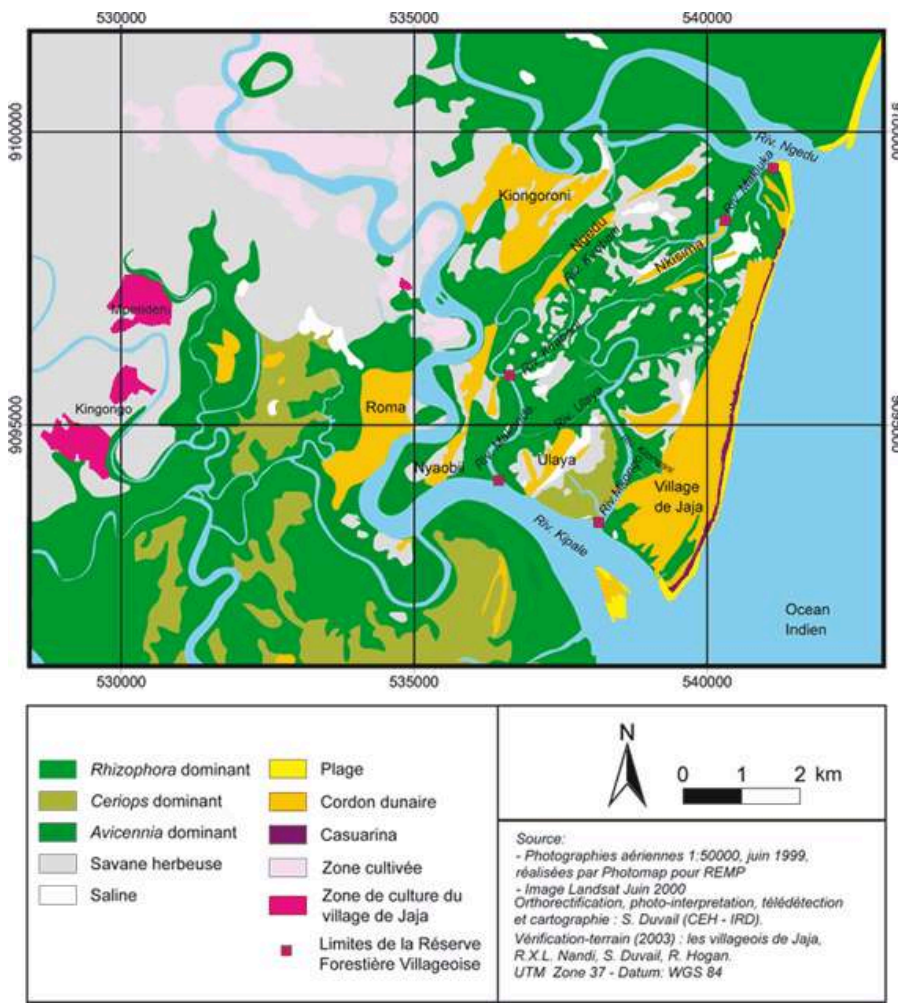

\section{Apports, limites et perspectives de l'exercice de cartographie participative}

\section{Du point de vue méthodologique}

L'utilisation d'images Landsat s'est avérée essentielle à la bonne représentation géométrique des grandes unités du paysage de type surfacique (terrasses, plaine inondable, mangrove) ou linéaire (pistes, cours d'eau). En revanche de telles images, d'une résolution de $30 \mathrm{~m}$, ne sont appropriées ni pour une description de la mosaïque de forêts, de savanes arborées et herbeuses, ni pour une description du parcellaire agricole. L'utilisation complémentaire de photographies aériennes et une analyse paysagère au sol, afin de vérifier et d'améliorer les classifications établies, se sont à cet égard révélées indispensables.

Le travail d'analyse paysagère a été réalisé par des équipes mixtes de villageois, chercheurs et gestionnaires. Une typologie a été produite sur la base d'une synthèse entre la typologie utilisée localement et la classification paysagère des botanistes et géographes. Cette combinaison entre savoirs locaux et techniques scientifiques s'est avérée très efficace en matière de cartographie et enrichissante pour les deux parties : pour les chercheurs, les classifications utilisées localement ont grandement facilité l'analyse paysagère. Un tel exercice de cartographie 'participative' permet également de mieux comprendre les modes de gestion du territoire et est un complément indispensable des entretiens sociologiques. Inversement, les villageois ont exprimé un 
grand intérêt à voir leur territoire cartographié et son étendue reconnue officiellement par les autorités locales.

Une légende commune a été produite à partir des différents types de paysages reconnus et représentés dans les quatre zones villageoises. Les quatre cartes assemblées représentent $16,5 \%$ de la surface administrative du district (fig. 1), cependant les analyses paysagères réalisées au cours de l'exercice de cartographie s'étendent au-delà des limites administratives des quatre villages et représentent environ $33 \%$ de la zone du district. Cet échantillonnage nous permettra de produire une cartographie des paysages à une échelle régionale.

Cette cartographie n'est cependant qu'une étape. Elle constitue une première description de l'occupation de l'espace villageois qui doit être complétée par une lecture plus anthropologique, tenant compte à la fois de la répartition spatiale des différents groupes sociaux, des règles d'attribution des terres et de gestion des ressources renouvelables, de l'utilisation économique des espaces et de leur valeur spirituelle. Il serait particulièrement intéressant d'analyser la perception de l'espace par les communautés villageoises, et notamment le rôle des sites sacrés dans la représentation du territoire des communautés.

En effet, l'exercice de cartographie a permis de mettre en valeur l'importance pour les communautés locales de petits autels dédiés aux esprits du lieu (forêt ou lac). Dans chaque village des points GPS ont été pris à la demande des habitants lorsque nous passions à proximité de ces sites sacrés. Le rôle joué par ces lieux d'offrandes dans la forme du territoire est une question qui mérite d'être approfondie. Il est probable que, comme dans beaucoup de sociétés africaines, une représentation topocentrique de l'espace prime sur la représentation géométrique occidentale (Le Roy, 1991). Il serait intéressant d'analyser, voire même de cartographier, cette représentation topocentrique de l'espace, en inventant des règles de représentation graphiques appropriées. Les différents modes de représentation (maquette, dessins) utilisés en préparation de l'exercice de cartographie offrent une première image de l'espace tel que perçu par les habitants. Ainsi, dans les villages de la vallée, l'espace est organisé linéairement autour des principaux cours d'eau et lacs, reflétant l'importance accordée au réseau hydrographique et aux inondations dans un système d'agriculture de décrue (fig. 6). Les forêts sacrées sont également représentées. Dans le delta, l'espace semble plutôt être organisé autour des îlots dunaires habitables (fig. 7). 
Figure 6 : Dessin du village de Mtanza-Msona Drawing of Mtanza-Msona village

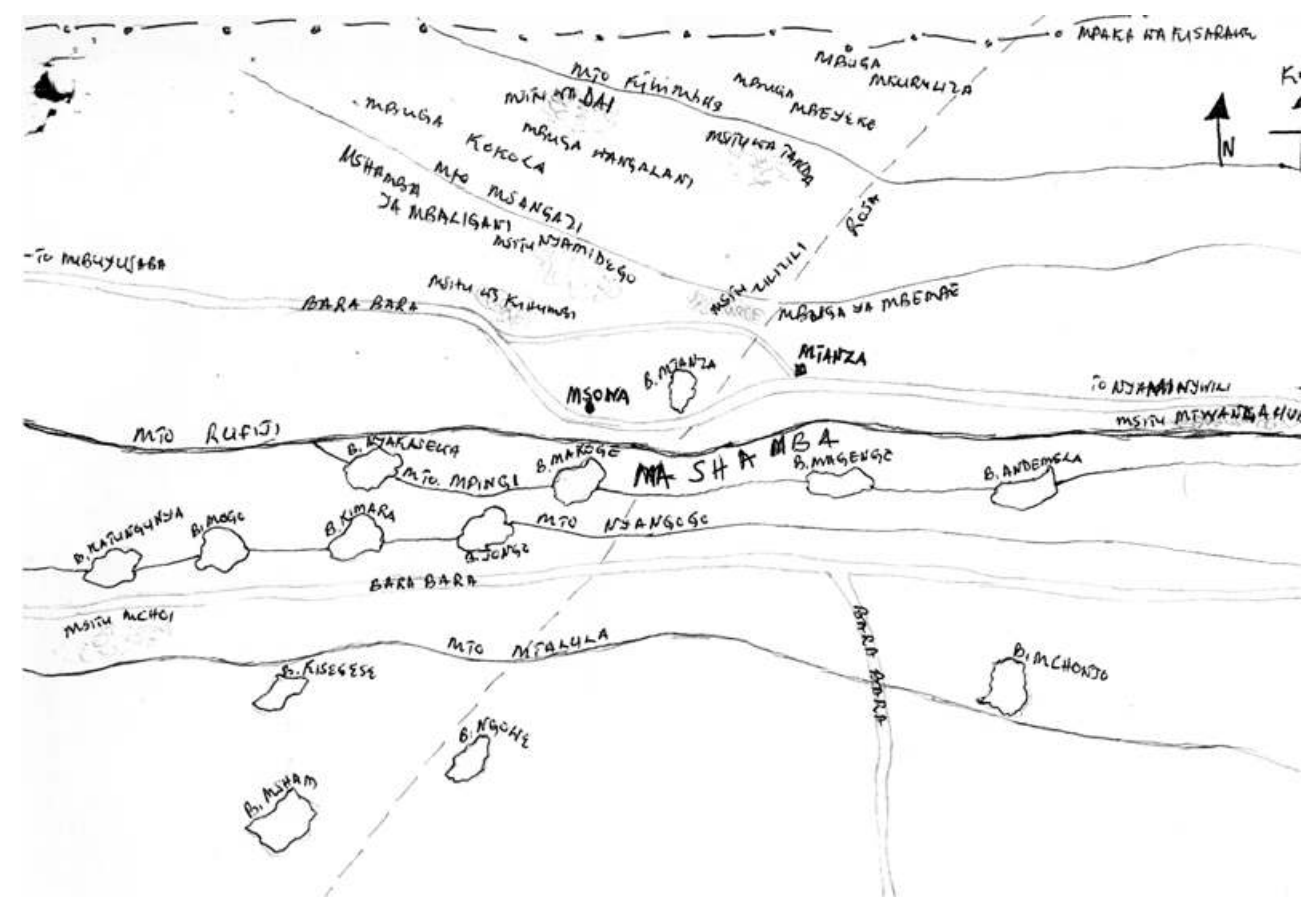

Figure 7 : Dessin du village de Twasalie Drawing of twasalie village

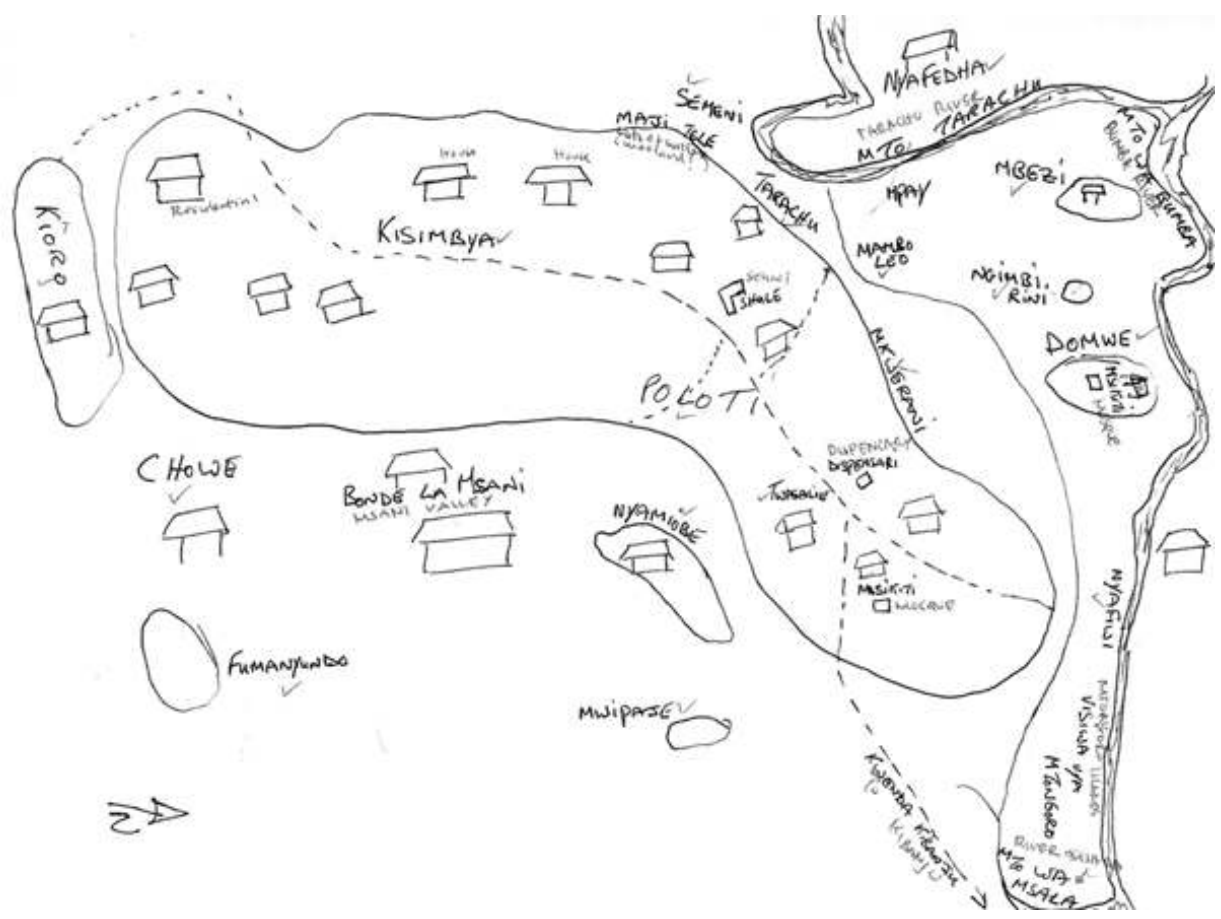

\section{Du point de vue de la gestion}

L'exercice de cartographie a facilité la compréhension mutuelle entre agents du district, villageois et scientifiques. 

villageoises de répondre à un besoin de reconnaissance légale de leur territoire fonctionnel. La nouvelle loi forestière qui permet au village de déclarer une partie de leur terroir en réserve forestière villageoise a été appliquée: le bornage et la cartographie des zones choisies comme réserves forestières villageoises a permis leur enregistrement officiel auprès de l'administration nationale. Concernant l'épineuse question des zones de culture traditionnelles, théoriquement interdites à la culture mais fortement cultivées dans les faits, un travail de cartographie fine a permis de dépasser la problématique d'une "occupation illégale» de la plaine inondable (telle qu'initialement perçue par les fonctionnaires du district). La pertinence de la plaine inondable comme zone de culture et la sophistication des techniques traditionnelles sont aujourd'hui admises par l'équipe du district. Le dialogue entamé entre les villageois et les agents du district porte à présent sur l'amélioration des conditions de culture dans la plaine inondable. La délimitation des territoires villageois a ainsi contribué à la reconnaissance des pratiques agricoles traditionnelles.

Plus généralement, la démarche de cartographie 'participative' a ouvert un cadre de négociation entre les différents acteurs. Même si le processus n'a pas toujours abouti à un consensus, comme dans le cas de la gestion des Lac Uba et Ruwe, une représentation spatiale commune du territoire villageois et la réflexion menée autour d'une planification à 10 ou 20 ans peut permettre de lisser, voire de prévenir des conflits éventuels entre lignages ou entre villages voisins. Dans le cas du conflit existant entre le village de Mtanza-Msona et la réserve de chasse voisine, le cadre formel a permis d'ouvrir une négociation, chose impossible lorsqu'elle avait été menée à la seule requête des villageois de Mtanza-Msona. des territoires administrés et a amélioré les relations district/villages, préalables nécessaires à une bonne mise en œuvre des politiques de décentralisation. La collaboration s'est faite autour de la constitution de bases de données pour les différents villages, élément non négligeable dans un contexte où l'information précise est rare. Deux agents du district ont été formés à l'utilisation du GPS, à la constitution de Systèmes d'Information Géographique et à leur mise à jour.

Le modèle plaît également aux bailleurs de fonds et ONG car il offre une méthodologie pour mettre en œuvre une co-gestion des ressources renouvelables. Ce processus de planification environnementale a remporté en 2004 le prix Equator Initiative du programme des Nations Unies, qui récompense les projets de développement durable contribuant à la lutte contre la pauvreté. 30000 dollars US ont été remis aux villages pour mettre en œuvre ces plans de gestion et fournir un conseil aux villages voisins désirant se doter d'un tel outil. Du fait de l'obtention de ce prix, le processus de planification environnementale a fait l'objet d'une large publicité dans les journaux tanzaniens et Est-africains. Le projet de Gestion de la Mangrove du Delta du Rufiji (Mangrove Management Project), soutenu par la coopération norvégienne, souhaite s'inspirer de l'expérience de REMP pour développer des plans de gestion villageois dans tous les villages du delta. Cette même méthode est mentionnée comme centrale dans le futur projet Seascape du WWF qui couvrira l'ensemble du Delta et les archipels de Mafia et de Songo-Songo. Enfin le projet PAMS (Partnership Actions for Mitigating Syndromes), sur financement de la coopération suisse, tente d'élargir l'expérience de gestion du terroir villageois à des écosystèmes partagés entre plusieurs villages. Deux sites pilotes 
ont été choisis, le Lac Zumbi (trois villages) et la forêt de Ngumburuni (quatre villages et la ville d'Ikwiriri), sites sur lesquels notre équipe a été sollicitée en matière de cartographie.

Au-delà de l'engouement actuel pour ce modèle, la généralisation d'un tel processus de co-gestion peut avoir un certain nombre de limites : s'il est réalisé de manière trop rapide et piloté de façon dirigiste par des organisations exogènes plutôt que correspondant à une véritable demande des communautés, il peut mener à un échec.

Il faut rappeler que l'identification d'une véritable demande, une analyse des relations de communication au sein du village, la définition d'un processus, l'établissement d'un plan de gestion a nécessité cinq années de négociation au sein du projet REMP et ce pour seulement quatre villages, préalablement sélectionnés. Un plan de gestion de l'environnement villageois réalisé dans la précipitation pourrait revenir à confier un pouvoir de gestion à des acteurs qui n'en ont pas l'autorité locale. Un tel processus nécessite donc que soit menée au préalable une analyse fine des relations de pouvoir entre les différents lignages composant le village. Il est également nécessaire que les facilitateurs de ce processus laissent au village le temps de trouver son propre arrangement institutionnel, sans l'imposer. Dans le cas contraire, un risque existe d'être confronté à une faible représentativité des acteurs émergeants, situation pouvant mener à des conflits en matière foncière ou de gestion des ressources renouvelables.

En effet, dans un contexte où les villages sont des créations artificielles de la politique d'Ujamaa, une réflexion préalable doit être menée sur la pertinence du concept de 'village', qui peut recouper des réalités très différentes (agglomération de populations migrantes, association de lignages amis, agrégation artificielle de lignages en mésentente comme dans le cas de Mbunju-Mvuleni). Dans tous les cas de figure, une étude des relations de pouvoir et d'autorité, une description précise de la situation foncière, des règles de gestion des ressources renouvelables, une description précise de l'organisation de l'espace doit accompagner voire précéder l'application des plans de gestion villageois.

47 Au-delà de ces problèmes de représentativité se pose également la question de la durabilité des transferts de gestion dans un contexte de libéralisme croissant. Ainsi, par exemple, la zone de chasse villageoise de Ngarambé-Tapika au sud du fleuve a été constituée sur le même principe d'un transfert de la gestion d'un secteur qui auparavant était le privilège de l'État, en l'occurrence de la faune. Lorsque sous gestion villageoise, la réserve de chasse a commencé à être rentable, sa gestion a alors été concédée par l'État à un opérateur de tourisme de chasse privé (Severe, 2003). Dans un tel scénario, le risque existe que les communautés locales soient lésées dans le partage des bénéfices. Le transfert de gestion est donc une démarche qui doit être accompagnée de moyens permettant au village d'imposer le respect du plan de gestion (organisation des patrouilles, moyen de locomotion, etc.) et d'un appui juridique pour s'opposer à des intérêts puissants.

\section{Conclusion}

Dans le cadre d'un processus de décentralisation des droits en matière de gestion forestière, une cartographie a été réalisée associant savoirs locaux et techniques scientifiques. Des cartes de l'occupation de l'espace ont été produites, précisant un 
agencement des paysages et une organisation de l'espace jusqu'ici mal connus des décideurs et des chercheurs.

La réalisation d'une carte du village s'est avérée un bon outil de dialogue entre les partenaires, facilitant la définition d'un plan de gestion environementale. Il convient cependant de relativiser l'opération de cartographie elle-même: sa véritable utilité réside dans le fait qu'elle sert de révélateur des enjeux de la gestion de l'espace. Pour résumer, il convient de garder à l'esprit que le processus (le dialogue) est plus important que les produits (le plan de gestion, la carte) qui doivent de toute façon être régulièrement mis à jour. Dans le contexte du Rufiji, cette mise à jour des produits se fera dans le cadre d'un partenariat entre le village, le district, et l'université de Dar Es Salaam. Se pose alors la question de la durabilité d'un tel processus, qui dépend de la qualité du partenariat. Si l'exercice de planification environnementale a favorisé un changement d'attitude de la part des agents du district envers les villageois et a permis une meilleure reconnaissance des pratiques de gestion locales, il doit cependant être accompagné d'une volonté politique de l'État de renforcer et de sécuriser les acquis d'une gestion décentralisée au niveau du village.

Sur le plan foncier, le bornage et la cartographie des zones choisies comme réserves forestières par les villages ont permis leur enregistrement officiel auprès de l'administration nationale. Plus généralement, dans un contexte de décalage entre le droit foncier coutumier et la loi étatique, une représentation de l'organisation de l'espace villageois, en stimulant une négociation entre les différents acteurs, a pu contribuer à clarifier une situation foncière confuse. Des mécanismes légaux sont cependant à mettre en place pour la résolution des conflits fonciers entre les différents villages, avec une définition du rôle médiateur que pourrait jouer le district en cas de conflit.

51 Une telle représentation "géométrique » de l'espace, telle que définie par E. Le Roy (1991), est requise par l'administration nationale pour l'enregistrement foncier des espaces forestiers choisis comme futures réserves forestières villageoises. Du point de vue strict de la planification économique au niveau villageois, il s'agit cependant d'un outil imparfait, qui prend mal en compte la dynamique des stratégies et pratiques des Warufiji, fortement liée aux variations des conditions hydro-climatiques. Une cartographie "géométrique » n'est pas non plus suffisamment informative des valeurs économiques et spirituelles que les populations accordent à ces espaces, ni des règles de gestion (intra- et inter-communautaire) des ressources renouvelables. Compte tenu du pouvoir juridique et symbolique de la carte, et pour éviter que la carte ne fige l'organisation spatiale sans restituer la valeur des différents espaces pour les populations locales, une analyse approfondie des espaces perçus, des enjeux fonciers et des règles de gestion des ressources renouvelables ainsi qu'une véritable description des stratégies et des pratiques des acteurs locaux en fonction des conditions hydroclimatiques sont essentielles et seront réalisées de 2005 à 2008, dans le cadre du projet de recherche de l'UR 169 de l'IRD. 


\section{BIBLIOGRAPHIE}

Agrar - und Hydrotechnik GMBH, 1982. - Irrigated agriculture development in the Lower Rufiji Valley, Prefeasibility study, Main report prepared for the Rufiji Basin Development Authority, United Republic of Tanzania, Essen (Allemagne), 228 p. + annexes

Baldus (R. D.), 2004. - « Lion Conservation in Tanzania leads to serious human-lion conflicts, with a case study of a man-eating lion killing 35 people », Tanzania Wildlife Discussion, $n^{\circ} 41$, Dar Es Salaam, GTZ Wildlife Programme in Tanzania, Wildlife Division, 39 p. + annexes et cartes.

Burgess (N. D.), Clarke (G. P.), 2000. - Coastal Forests of Eastern Africa, Gland/Cambridge, IUCN, 443 p.

DURAND (J.-M.), 2003. - « Implementation of the Rufiji Forest Action Plan With Special Emphasis on Community Based Natural Resources Management and a Case study of Ngumburuni Forest ", REMP Technical Reports, $n^{\circ}$ 45, 168 p. [http://www.iucn.org/themes/wetlands/REMP.html].

HUNTING TECHNICAL SERVICES, 1996. - National Reconnaissance Level Land Use and Natural resources Map, 2 cartes (Feuilles Utete et Mafia, échelle 1:250 000).

JAJA VILLAGE, 2004. - Our Village Environmental Management plan : an account of how we drew it up and are implementing it, Nairobi (Kenya), IUCN/Eastern Africa Regional Programme, version anglaise, traduite du swahili, 54 p. [http://www.iucn.org/themes/wetlands/REMP/VEMPS/jaja.pdf].

JOHN (P.), HAMERLYNCK (O.), 2003. - Collaborative Forest Management: the Rufiji District Experience, Participatory Forest Management Practitioners and Facilitators Meeting, Morogoro (Tanzanie) 8-10 July 2003, $18 \mathrm{p}$.

LE ROY (E.), 1991. - « Introduction Générale », dans LE BRIS (E.), LE ROY (E.), MATHIEU (P.),

L'appropriation de la terre en Afrique noire, Manuel d'analyse, de décision et de gestion foncière, Paris, Karthala, p. 11-23.

MAJAMBA (H.), MACHA (T.), MSHANA (E.), 2001. - « Training of Villagers in Legal Issues Pertaining to Community Control of Natural Resources ", REMP Technical Report, $\mathrm{n}^{\circ} 21,20 \mathrm{p}$.

MASALU (D. C. P.), 2003. - « Challenges of coastal area management in coastal developing countries - lessons from the proposed Rufiji delta prawn farming project, Tanzania », Ocean \& Coastal Management, 46, p. 175-188.

MBUNJU-MVULENI VILLAGE, 2004. - Our Village Environmental Management plan : an account of how we drew it up and are implementing it, IUCN-Eastern Africa Regional Programme, Nairobi (Kenya), version anglaise, traduite du swahili, 62 p. [http://www.iucn.org/themes/wetlands/REMP/ VEMPS/Mbuju.pdf].

MILLEDGE (S. A. H.), KAALE (B. K.), 2004. - Bridging the gap: Linking timber trade with infrastructutral development and poverty eradication efforts in southern Tanzania, Dar Es Salaam, TRAFFIC (The Wildlife Trade Monitoring Network), 126 p.

MNIWASA (E.), SHAURI (V.), 2001. - Review of the decentralization process and it's impact on environmental and natural resources management in Tanzania, LEAT (Lawyers' Environmental Action Team), Dar Es Salaam (Tanzanie), $27 \mathrm{p}$.

MTANZA-MSONA VILLAGE, 2004. - Our Village Environmental Management plan : an account of how we drew it up and are implementing it, Nairobi, IUCN/Eastern Africa Regional Programme, version anglaise, traduite du swahili, 54 p. [http://www.iucn.org/themes/wetlands/REMP/VEMPS/mtanza.pdf]. 
MWILAWA (A. J.), 2003. - « Assessment of potential rangeland resources in selected areas towards designing a livestock development strategy in Rufiji District », REMP Technical Report, $\mathrm{n}^{\circ}$ 40, $42 \mathrm{p}$. [http://www.iucn.org/themes/wetlands/REMP.html].

NATIONAL BUREAU OF STATISTICS, 2003. - Population and housing Census of 2002, United Republic of Tanzania, n.p.

RUFIJI DISTRICT COUNCIL, 1997. - Rufiji District, Socio-economic Profile, United Republic of Tanzania, 72 p.

SEVERE (E. M. L.), 2003. - Community Tourism Gateway to Poverty Reduction, IIPT, $2^{\text {nd }}$ African

Conference on Peace through Tourism, 15 p.

TANZANIA'S NATURAL RESOURCES INFORMATION CENTRE, 2001. - Map of the Rufiji District (scale 1:50 000), Tanzania's Natural Resources Information Centre, University of Dar Es Salaam for REMP-IUCN.

TELFORD (A. M.), 1929. - Report on the development of the Rufiji and Kilombero Valley, Londres, Crown Agents for the Colonies, 42 p. + annexes et cartes.

TURPIE (J.), 2000. - « The use and value of natural resources of the Rufiji Floodplain and Delta ", IUCN REMP Technical Reports, n 17, 77 p. + annexes [http://www.iucn.org/themes/wetlands/ REMP.html].

TWASALIE VILLAGE, 2004. - Our Village Environmental Management plan : an account of how we drew it up and are implementing it, Nairobi, IUCN-Eastern Africa Regional Programme, version anglaise, traduite du swahili, 60 p. [http://www.iucn.org/themes/wetlands/REMP/VEMPS/Twasalie.pdf].

\section{NOTES}

1. Ce terme est employé pour désigner les différentes ethnies de la basse vallée du Rufiji (en majorité d'origine Ngendereko et Nyagatwa).

2. Notamment Dalbergia melanoxylon 'Mpingo', Milicia exelsa 'Mvule', Khaya anthotheca 'Mkangazi', Newtonia species, 'Mdadarika' Pterocarpus angolensis 'Mninga', Afzelia quanzensis 'Mkongo', Millettia stuhlmannii ‘Mpangapanga' et Swartzia madagascarensis 'Msekeseke'.

3. Les photographies ont été acquises par Photomap Ltd. (Nairobi, Kenya) pour le compte du projet

\section{RÉSUMÉS}

Comme dans d'autres pays en Afrique, les nouvelles lois de décentralisation des services de l'État tanzanien permettent aux communautés locales de gérer leurs ressources renouvelables à l'échelle villageoise. Le Rufiji Environnemental Management Project a accompagné pendant 5 années un processus de transfert des droits de gestion des ressources renouvelables, notamment forestières dans 4 villages du District du Rufiji, dans le Sud de la Tanzanie. Des plans de gestion des villages ont été définis, comprenant des cartes précisant l'organisation de l'espace et l'agencement des paysages, produites sur la base d'images Landsat, de photographies aériennes et d'analyses paysagères réalisées par des équipes multi-institutionnelles. La représentation 
cartographique et la planification environnementale, réalisées de façon participative, sont des outils qui favorisent le dialogue entre populations locales, gestionnaires de l'État et chercheurs. Le bornage et la cartographie des zones choisies comme réserves forestières villageoises a permis leur enregistrement officiel auprès de l'administration nationale. Plus généralement, dans un contexte de décalage entre le droit foncier coutumier et la loi étatique, une représentation de l'organisation de l'espace villageois, en stimulant une négociation entre les différents acteurs, peut contribuer à clarifier une situation foncière confuse. L'exercice de planification environnementale peut aussi favoriser un développement économique local mais est à compléter par une analyse détaillée de la valeur économique et spirituelle des différents espaces et par une description des règles de gestion des ressources renouvelables. Il doit également être accompagné d'une volonté politique de l'État de renforcer et de sécuriser les acquis de cette gestion décentralisée.

As in other countries in Africa, the new Tanzanian legislation on decentralisation allows communities to locally manage their natural resources. From 1998 to 2003, the Rufiji Environmental Management Project promoted such a transfer of authority, from the central government to 4 villages of the Rufiji District in southern Tanzania, mostly for forest resources. Villages Environmental Management Plans (VEMP) have been designed by the local communities. Land-use maps have been produced by multi-institutional teams using Landsat images, aerial photographs, detailed landscape analysis and ground-truthing. Cartography and Environmental Management, carried out in a participatory way, were shown to be efficient tools for the improvement of the communication between local populations, government institutions and researchers. The mapping of 4 forest areas, chosen by the villagers, was instrumental in their official recognition as Village Forest Reserves. More generally, a common representation of land uses in the Rufiji District, by stimulating a negotiation between the stakeholders can contribute to clarify a fuzzy land-tenure situation. But for improved planning, this participatory land use mapping exercise has to be complemented by a detailed analysis of the economic and spiritual values of the different landscape components, and by a description of the local rules of resource sharing. The drive towards increased local management of natural resources still needs to be supported by a strong Government will to empower and to secure the local benefits of the decentralisation process.

\section{INDEX}

Mots-clés : cartographie, décentralisation, foncier, gestion des ressources

Keywords : cartography, decentralisation, land tenure, resource management Index géographique : Tanzanie, Rufiji (district)

\section{AUTEURS}

\section{STÉPHANIE DUVAIL}

IRD - MNHN - UR 169, "Patrimoines naturels, territoires et identités (PATIS)", USM 102, Case postale $n^{\circ} 26,57$ rue Cuvier - 75231 Paris cedex 05, France, stephanie.duvail@ird.fr

\section{ROSE HOGAN}

Rufiji Environmental Management project - IUCN, Dar Es Salaam, Tanzanie, rhog@eircom.net 


\section{PILI MWAMBESO}

Rufiji District Council - Agriculture Department, Utete, Tanzanie, rempute1@bushmail.net

REVOCATUS XL NANDI

Rufiji District Council - Agriculture Department, Utete, Tanzanie,

rempute1@bushmail.net

\section{RICHARD ELIBARIKI}

Rufiji Environmental Management project - IUCN, Dar Es Salaam, Tanzanie, relibariki@yahoo.co.uk

\section{OLIVIER HAMERLYNCK}

Rufiji Environmental Management project - IUCN, Dar Es Salaam, Tanzanie, olivier.hamerlynck@wanadoo.fr 